\title{
On Appell-type Changhee polynomials and numbers
}

\author{
Jeong Gon Lee ${ }^{1}$, Lee-Chae Jang ${ }^{2 *}$, Jong-Jin Seo ${ }^{3}$, Sang-Ki Choi ${ }^{4}$ and Hyuck In Kwon ${ }^{5}$
}

${ }^{*}$ Correspondence:

lcjang@konkuk.ac.kr

${ }^{2}$ Graduate School of Education,

Konkuk University, Seoul, 143-701,

Republic of Korea

Full list of author information is

available at the end of the article

\section{Abstract}

In this paper, we consider the Appell-type Changhee polynomials and derive some properties of these polynomials. Furthermore, we investigate certain identities for these polynomials.

MSC: 05A10; 11B68; 11S80; 05A19

Keywords: Changhee polynomials; Appell-type Changhee polynomials; degenerate Bernoulli polynomials; beta functions

\section{Introduction}

Let $p$ be a fixed odd prime number. Throughout this paper, we denote by $\mathbb{Z}_{p}, \mathbb{Q}_{p}$, and $\mathbb{C}_{p}$ the ring of $p$-adic integers, the field of $p$-adic numbers, and the completion of algebraic closure of $\mathbb{Q}_{p}$. The $p$-adic norm $|\cdot|_{p}$ is normalized as $|p|_{p}=\frac{1}{p}$. Let $C\left(\mathbb{Z}_{p}\right)$ be the space of continuous functions on $\mathbb{Z}_{p}$. For $f \in C\left(\mathbb{Z}_{p}\right)$, the fermionic $p$-adic integral on $\mathbb{Z}_{p}$ is defined by Kim to be

$$
I_{-1}(f)=\int_{\mathbb{Z}_{p}} f(x) d \mu_{-1}(x)=\lim _{N \rightarrow \infty} \sum_{x=0}^{p^{N}-1} f(x)(-1)^{x}
$$

(see [1-19]). For $f_{1}(x)=f(x+1)$, we have

$$
I_{-1}\left(f_{1}\right)+I_{-1}(f)=2 f(0) \text {. }
$$

As is well known, the Changhee polynomials are defined by the generating function

$$
\int_{\mathbb{Z}_{p}}(1+t)^{x+y} d \mu_{-1}(y)=\frac{2}{2+t}(1+t)^{x}=\sum_{n=0}^{\infty} \mathrm{Ch}_{n}(x) \frac{t^{n}}{n !}
$$

When $x=0, \mathrm{Ch}_{n}=\mathrm{Ch}_{n}(0)$ are called the Changhee numbers (see $\left.[17,18,20]\right)$. The gamma and beta functions are defined by the following definite integrals: for $\alpha>0, \beta>0$,

$$
\Gamma(\alpha)=\int_{0}^{\infty} e^{-t} t^{\alpha-1} d t
$$

(c) 2016 Lee et al. This article is distributed under the terms of the Creative Commons Attribution 4.0 International License (http://creativecommons.org/licenses/by/4.0/), which permits unrestricted use, distribution, and reproduction in any medium, provided you give appropriate credit to the original author(s) and the source, provide a link to the Creative Commons license, and indicate if changes were made. 
and

$$
\begin{aligned}
B(\alpha, \beta) & =\int_{0}^{1} t^{\alpha-1}(1-t)^{\beta-1} d t \\
& =\int_{0}^{\infty} \frac{t^{\alpha-1}}{(1+t)^{\alpha+\beta}} d t
\end{aligned}
$$

(see[20, 21]). Thus, by (4) and (5) we have

$$
\Gamma(\alpha+1)=\alpha \Gamma(\alpha), \quad B(\alpha, \beta)=\frac{\Gamma(\alpha) \Gamma(\beta)}{\Gamma(\alpha+\beta)}
$$

Stirling numbers of the first kind are defined by

$$
(\log (1+t))^{n}=n ! \sum_{m=n}^{\infty} S_{1}(m, n) \frac{t^{m}}{m !}
$$

and the Stirling numbers of the second kind are defined by

$$
\left(e^{t}-1\right)^{n}=n ! \sum_{l=n}^{\infty} S_{2}(n, l) \frac{t^{l}}{l !} \quad(n \geq 0) .
$$

Recently, Lim and Qi [20] have derived integral identities for Appell-type $\lambda$-Changhee numbers from the fermionic integral equation. The degenerate Bernoulli polynomials, a degenerate version of the well-known family of polynomials, were introduced by Carlitz, and after that, many researchers have studied the degenerate special polynomials (see [1$3,20,22-28])$.

The goal of this paper is to consider the Appell-type Changhee polynomials, another version of the Changhee polynomials in (3), and derive some properties of these polynomials. Furthermore, we investigate certain identities for these polynomials.

\section{Some identities for Appell-type Changhee polynomials}

Now we define the Appell-type Changhee polynomials $\mathrm{Ch}_{n}^{*}(x)$ by

$$
\frac{2}{2+t} e^{x t}=\sum_{n=0}^{\infty} \operatorname{Ch}_{n}^{*}(x) \frac{t^{n}}{n !}
$$

When $x=0$, the Changhee numbers $\mathrm{Ch}_{n}^{*}=\mathrm{Ch}_{n}^{*}(0)$ are equal to the Changhee numbers $\mathrm{Ch}_{n}=\mathrm{Ch}_{n}(0)$. From (9) we have

$$
\begin{aligned}
\frac{2}{2+t} e^{x t} & =\left(\sum_{m=0}^{\infty} \mathrm{Ch}_{m}^{*} \frac{t^{m}}{m !}\right)\left(\sum_{l=0}^{\infty} x^{l} \frac{t^{l}}{l !}\right) \\
& =\sum_{n=0}^{\infty}\left(\sum_{m=0}^{n}\left(\begin{array}{c}
n \\
m
\end{array}\right) \mathrm{Ch}_{m}^{*} x^{n-m}\right) \frac{t^{n}}{n !} .
\end{aligned}
$$

By (10) we have the following theorem. 
Theorem 1 For $n \in \mathbb{N}$, we have

$$
\mathrm{Ch}_{n}^{*}(x)=\sum_{m=0}^{n}\left(\begin{array}{l}
n \\
m
\end{array}\right) \mathrm{Ch}_{m}^{*} x^{n-m}
$$

By (9), replacing $t$ by $e^{t}-1$, we get

$$
\frac{2}{2+e^{t}-1} e^{x\left(e^{t}-1\right)}=\sum_{n=0}^{\infty} \operatorname{Ch}_{n}^{*}(x) \frac{\left(e^{t}-1\right)^{n}}{n !}
$$

Then we have

$$
\begin{aligned}
\mathrm{RHS} & =\sum_{n=0}^{\infty} \mathrm{Ch}_{n}^{*}(x) \frac{\left(e^{t}-1\right)^{n}}{n !} \\
& =\sum_{n=0}^{\infty} \mathrm{Ch}_{n}^{*}(x) \frac{1}{n !} n ! \sum_{l=n}^{\infty} S_{2}(l, n) \frac{t^{l}}{l !} \\
& =\sum_{l=0}^{\infty} \sum_{n=0}^{l} \mathrm{Ch}_{n}^{*}(x) S_{2}(l, n) \frac{t^{l}}{l !},
\end{aligned}
$$

where $S_{2}(l, n)$ are the Stirling numbers of the second kind, and

$$
\begin{aligned}
\text { LHS } & =\frac{2}{1+e^{t}} e^{x\left(e^{t}-1\right)} \\
& =\sum_{m=0}^{\infty} E_{m} \frac{t^{m}}{m !} \sum_{n=0}^{\infty} \operatorname{Bel}_{n}(x) \frac{t^{n}}{n !} \\
& =\sum_{l=0}^{\infty} \sum_{n=0}^{l}\left(\begin{array}{l}
l \\
n
\end{array}\right) E_{n} \operatorname{Bel}_{l-n}(x) \frac{t^{l}}{l !} .
\end{aligned}
$$

It is well known that the Bell polynomials are defined by the generating function

$$
e^{x\left(e^{t}-1\right)}=\sum_{n=0}^{\infty} \operatorname{Bel}_{n}(x) \frac{t^{n}}{n !}
$$

(see [8]). By (13) and (14) we have the following theorem.

Theorem 2 For $l \in \mathbb{N}$, we have

$$
\sum_{n=0}^{l} \mathrm{Ch}_{n}^{*}(x) S_{2}(l, n)=\sum_{n=0}^{l}\left(\begin{array}{l}
l \\
n
\end{array}\right) E_{n} \operatorname{Bel}_{l-n}(x) .
$$

By (11) we can derive the following equation:

$$
\begin{aligned}
\frac{d}{d x} \mathrm{Ch}_{n}^{*}(x) & =\sum_{m=0}^{n-1}\left(\begin{array}{c}
n \\
m
\end{array}\right) \mathrm{Ch}_{m}^{*}(n-m) x^{n-m-1} \\
& =n \mathrm{Ch}_{n-1}^{*}(x) .
\end{aligned}
$$


From (16) we get

$$
\begin{aligned}
n \int_{0}^{x} \mathrm{Ch}_{n-1}^{*}(s) d s & =\int_{0}^{x} \frac{d}{d s} \operatorname{Ch}_{n}^{*}(s) d s \\
& =\left.\mathrm{Ch}_{n}^{*}(s)\right|_{s=0} ^{x} \\
& =\mathrm{Ch}_{n}^{*}(x)-\mathrm{Ch}_{n}^{*}
\end{aligned}
$$

By (17) we can derive the following theorem.

Theorem 3 For $n \in \mathbb{N}$, we have

$$
\frac{\mathrm{Ch}_{n+1}^{*}(x)-\mathrm{Ch}_{n+1}^{*}}{n+1}=\int_{0}^{x} \mathrm{Ch}_{n}^{*}(s) d s .
$$

By (4) we note that

$$
\begin{aligned}
2 & =\left(\sum_{n=0}^{\infty} \mathrm{Ch}_{n}^{*} \frac{t^{n}}{n !}\right)(2+t) \\
& =\left(\sum_{n=0}^{\infty} 2 \mathrm{Ch}_{n}^{*} \frac{t^{n}}{n !}\right)+t \sum_{n=0}^{\infty} \mathrm{Ch}_{n}^{*} \frac{t^{n}}{n !} \\
& =\left(\sum_{n=0}^{\infty} 2 \mathrm{Ch}_{n}^{*} \frac{t^{n}}{n !}\right)+\sum_{n=1}^{\infty} n \mathrm{Ch}_{n-1}^{*} \frac{t^{n}}{n !} \\
& =2 \mathrm{Ch}_{0}^{*}+\sum_{n=1}^{\infty}\left(2 \mathrm{Ch}_{n}^{*}+n \mathrm{Ch}_{n-1}^{*}\right) \frac{t^{n}}{n !} .
\end{aligned}
$$

By (19) we have the following theorem.

Theorem 4 For $n \in \mathbb{N}$, we have

$$
\mathrm{Ch}_{0}^{*}=1, \quad 2 \mathrm{Ch}_{n}^{*}+n \mathrm{Ch}_{n-1}^{*}=0 \quad \text { if } n \geq 1 .
$$

Now we observe that

$$
\begin{aligned}
\sum_{n=0}^{\infty} \operatorname{Ch}_{n}^{*}(1-x) \frac{t^{n}}{n !} & =\frac{2}{2+t} e^{(1-x) t} \\
& =\frac{2}{2+t} e^{t} e^{-x t} \\
& =\left(\sum_{l=0}^{\infty} \operatorname{Ch}_{l}^{*}(1) \frac{t^{l}}{l !}\right)\left(\sum_{m=0}^{\infty}(-x)^{m} \frac{t^{m}}{m !}\right) \\
& =\sum_{n=0}^{\infty}\left(\sum_{m=0}^{n}\left(\begin{array}{c}
n \\
m
\end{array}\right) \mathrm{Ch}_{n-m}^{*}(1)(-x)^{m}\right) \frac{t^{n}}{n !}
\end{aligned}
$$

From (21) we obtain the following theorem. 
Theorem 5 For $n \in \mathbb{N}$, we have

$$
\mathrm{Ch}_{n}^{*}(1-x)=\sum_{m=0}^{n}\left(\begin{array}{l}
n \\
m
\end{array}\right) \mathrm{Ch}_{n-m}^{*}(1)(-x)^{m}
$$

By (22) we get

$$
\begin{aligned}
\int_{0}^{1} \mathrm{Ch}_{n}^{*}(1-x) x^{n} d x & =\sum_{m=0}^{n}\left(\begin{array}{l}
n \\
m
\end{array}\right) \mathrm{Ch}_{n-m}^{*}(1)(-1)^{m} \int_{0}^{1} x^{n+m} d x \\
& =\sum_{m=0}^{n}\left(\begin{array}{l}
n \\
m
\end{array}\right)(-1)^{m} \frac{\mathrm{Ch}_{n-m}^{*}(1)}{n+m+1} .
\end{aligned}
$$

From (16) we note that

$$
\begin{aligned}
\int_{0}^{1} y^{n} \mathrm{Ch}_{n}^{*}(x+y) d y & \left.y^{n+1} \mathrm{Ch}_{n}^{*}(x+y)\right|_{y=0} ^{1}-\frac{1}{n+1} \int_{0}^{1} y^{n+1} \frac{d}{d y} \mathrm{Ch}_{n}^{*}(x+y) d y \\
= & \frac{\mathrm{Ch}_{n}^{*}(x+1)}{n+1}-\frac{n}{n+1} \int_{0}^{1} y^{n+1} \mathrm{Ch}_{n-1}^{*}(x+y) d y \\
= & \frac{\mathrm{Ch}_{n}^{*}(x+1)}{n+1}-\frac{n}{n+1}\left(\left.\frac{\mathrm{Ch}_{n-1}^{*}(x+y)}{n+2} y^{n+2}\right|_{y=0} ^{1}\right) \\
& +(-1)^{2} \frac{n}{n+1} \frac{1}{n+2}(n-1) \int_{0}^{1} y^{n+2} \mathrm{Ch}_{n-2}^{*}(x+y) d y \\
= & \frac{\mathrm{Ch}_{n}^{*}(x+1)}{n+1}-\frac{n}{n+1} \frac{\mathrm{Ch}_{n-1}^{*}(x+1)}{n+2}+(-1)^{2} \frac{n}{n+1} \frac{n-1}{n+2} \int_{0}^{1} y^{n+2} \mathrm{Ch}_{n-2}^{*}(x+y) d y \\
= & \frac{\mathrm{Ch}_{n}^{*}(x+1)}{n+1}-\frac{n}{n+1} \frac{\mathrm{Ch}_{n-1}^{*}(x+1)}{n+2}+(-1)^{2} \frac{n}{n+1} \frac{n-1}{n+2} \frac{\mathrm{Ch}_{n-2}^{*}(x+1)}{n+3} \\
& +(-1)^{3} \frac{n}{n+1} \frac{n-1}{n+2} \frac{n-2}{n+3} \int_{0}^{1} y^{n+3} \mathrm{Ch}_{n-3}^{*}(x+y) d y .
\end{aligned}
$$

Also, we get

$$
\int_{0}^{1} y^{2 n-1} \mathrm{Ch}_{1}^{*}(x+y) d y=\left.\frac{\mathrm{Ch}_{1}^{*}(x+y)}{2 n} y^{2 n}\right|_{y=0} ^{1}-\frac{1}{2 n} \int_{0}^{1} y^{2 n} \mathrm{Ch}_{0}^{*}(x+y) d y .
$$

From (11) we get

$$
\mathrm{Ch}_{0}^{*}(x)=1,
$$

and hence

$$
\begin{aligned}
\int_{0}^{1} y^{2 n-1} \mathrm{Ch}_{1}^{*}(x+y) d y & =\frac{\mathrm{Ch}_{1}^{*}(x)}{2 n}-\frac{1}{2 n} \int_{0}^{1} y^{2 n} d y \\
& =\frac{\mathrm{Ch}_{1}^{*}(x)}{2 n}-\frac{1}{2 n(2 n+1)} .
\end{aligned}
$$


By (27), continuing the process in (24), we have

$$
\begin{aligned}
& \int_{0}^{1} y^{n} \mathrm{Ch}_{n}^{*}(x+y) d y \\
& \quad=\frac{\mathrm{Ch}_{n}^{*}(x+1)}{n+1}+\sum_{m=1}^{n}(-1)^{m} \mathrm{Ch}_{n-m}^{*}(x+1) \frac{n(n-1) \cdots(n-m+1)}{(n+1)(n+2) \cdots(n+m+1)}
\end{aligned}
$$

We note that

$$
\begin{aligned}
\mathrm{Ch}_{n}^{*}(x+y) & =\mathrm{Ch}_{n}^{*}(x+1+y-1) \\
& =\sum_{l=1}^{n}\left(\begin{array}{l}
n \\
l
\end{array}\right) \mathrm{Ch}_{l}^{*}(x+1)(-1)^{n-l}(1-y)^{n-l} .
\end{aligned}
$$

By (29) we get

$$
\begin{aligned}
\int_{0}^{1} & y^{n} \mathrm{Ch}_{n}^{*}(x+y) d y \\
& =\sum_{l=1}^{n}\left(\begin{array}{l}
n \\
l
\end{array}\right) \mathrm{Ch}_{l}^{*}(x+1)(-1)^{n-l} \int_{0}^{1} y^{n}(1-y)^{n-l} d y \\
& =\sum_{l=1}^{n}\left(\begin{array}{l}
n \\
l
\end{array}\right) \mathrm{Ch}_{l}^{*}(x+1)(-1)^{n-l} B(n+1, n-l+1) \\
& =\sum_{l=0}^{n}\left(\begin{array}{l}
n \\
l
\end{array}\right) \mathrm{Ch}_{l}^{*}(x+1)(-1)^{n-l} \frac{\Gamma(n+1) \Gamma(n-l+1)}{\Gamma(2 n-l+2)} \\
& =\sum_{l=0}^{n}(-1)^{n-l}\left(\begin{array}{c}
n \\
l
\end{array}\right) \frac{n !(n-l) !}{(2 n-l+1) !} \mathrm{Ch}_{l}^{*}(x+1) \\
& =\sum_{l=0}^{n}(-1)^{n-l} \frac{n\left(\begin{array}{c}
n \\
l
\end{array}\right)}{(2 n-l+1)\left(\begin{array}{c}
2 n-l \\
n
\end{array}\right)} \mathrm{Ch}_{l}^{*}(x+1) .
\end{aligned}
$$

By (28) and (30) we have the following theorem.

Theorem 6 For $n \in \mathbb{N}$, we have

$$
\begin{aligned}
& \sum_{l=0}^{n}(-1)^{n-l} \frac{n\left(\begin{array}{c}
n \\
l
\end{array}\right)}{(2 n-l+1)\left(\begin{array}{c}
2 n-l \\
n
\end{array}\right)} \mathrm{Ch}_{l}^{*}(x+1) \\
& \quad=\frac{\mathrm{Ch}_{n}^{*}(x+1)}{n+1}+\sum_{m=1}^{n}(-1)^{m} \mathrm{Ch}_{n-m}^{*}(x+1) \frac{n(n-1) \cdots(n-m+1)}{(n+1)(n+2) \cdots(n+m+1)}
\end{aligned}
$$

From (16) we note that

$$
\begin{aligned}
& \int_{0}^{1} y^{n} \mathrm{Ch}_{n}^{*}(x+y) d y \\
& \quad=\left.\frac{\mathrm{Ch}_{n+1}^{*}(x+y)}{n+1} y^{n}\right|_{y=0} ^{1}-\frac{1}{n+1} n \int_{0}^{1} y^{n-1} \mathrm{Ch}_{n+1}^{*}(x+y) d y
\end{aligned}
$$




$$
\begin{aligned}
= & \frac{\mathrm{Ch}_{n+1}^{*}(x+1)}{n+1}-\frac{n}{n+1} \int_{0}^{1} y^{n-1} \mathrm{Ch}_{n+1}^{*}(x+y) d y \\
= & \frac{\mathrm{Ch}_{n+1}^{*}(x+1)}{n+1}-\frac{n}{n+1} \frac{\mathrm{Ch}_{n+2}^{*}(x+1)}{n+2}+\frac{n(n-1)}{(n+1)(n+2)} \int_{0}^{1} y^{n-2} \mathrm{Ch}_{n+2}^{*}(x+y) d y \\
= & \frac{\mathrm{Ch}_{n+1}^{*}(x+1)}{n+1}-\frac{n}{n+1} \frac{\mathrm{Ch}_{n+2}^{*}(x+1)}{n+2}+\frac{n(n-1)}{(n+1)(n+2)} \frac{\mathrm{Ch}_{n+3}^{*}(x+1)}{n+3} \\
& -\frac{n(n-1)(n-2)}{(n+1)(n+2)(n+3)} \int_{0}^{1} y^{n-3} \mathrm{Ch}_{n+3}^{*}(x+y) d y .
\end{aligned}
$$

Also, we have

$$
\begin{aligned}
& \int_{0}^{1} y \mathrm{Ch}_{2 n-1}^{*}(x+y) d y \\
& =\left.\frac{\mathrm{Ch}_{2 n}^{*}(x+y)}{2 n} y\right|_{y=0} ^{1}-\frac{1}{2 n} \int_{0}^{1} 1 \cdot \mathrm{Ch}_{2 n}^{*}(x+y) d y \\
& =\frac{\mathrm{Ch}_{2 n}^{*}(x+1)}{2 n}-\left.\frac{1}{2 n} \frac{1}{2 n+1} \mathrm{Ch}_{2 n+1}^{*}(x+y)\right|_{y=0} ^{1} \\
& =\frac{\mathrm{Ch}_{2 n}^{*}(x+1)}{2 n}-\frac{\mathrm{Ch}_{2 n+1}^{*}(x+1)-\mathrm{Ch}_{2 n+1}^{*}(x)}{2 n(2 n+1)}
\end{aligned}
$$

By (30), continuing the process in (28), we obtain the following theorem.

Theorem 7 For $n \in \mathbb{N}$, we have

$$
\begin{aligned}
\sum_{l=0}^{n}(-1)^{n-l} \frac{n\left(\begin{array}{l}
n \\
l
\end{array}\right)}{(2 n-l+1)\left(\begin{array}{c}
2 n-l \\
n
\end{array}\right)} \mathrm{Ch}_{l}^{*}(x+1) \\
=\frac{\mathrm{Ch}_{n+1}^{*}(x+1)}{n+1}+\sum_{m=1}^{n-1}(-1)^{m} \mathrm{Ch}_{n+m+1}^{*}(x+1) \frac{n(n-1) \cdots(n-m+1)}{(n+1)(n+2) \cdots(n+m+1)} \\
\quad+(-1)^{n} \frac{n !}{(2 n+1)_{n+1}}\left(\mathrm{Ch}_{2 n+1}^{*}(x+1)-\mathrm{Ch}_{2 n+1}^{*}(1)\right) .
\end{aligned}
$$

Now, we have

$$
\begin{aligned}
\int_{0}^{1} \mathrm{Ch}_{n}^{*}(x) \mathrm{Ch}_{m}^{*}(x) d x \\
=\frac{\left.\mathrm{Ch}_{n+1}^{*}(x) \mathrm{Ch}_{m}^{*}(x)\right|_{0} ^{1}-\frac{1}{n+1} m \int_{0}^{1} \mathrm{Ch}_{n+1}^{*}(x) \mathrm{Ch}_{m-1}^{*}(x) d x}{=} \frac{1}{n+1}\left(\mathrm{Ch}_{n+1}^{*}(1) \mathrm{Ch}_{m}^{*}(1)-\mathrm{Ch}_{n+1}^{*}(0) \mathrm{Ch}_{m}^{*}(0)\right) \\
\quad-\frac{m}{n+1} \int_{0}^{1} \mathrm{Ch}_{n+1}^{*}(x) \mathrm{Ch}_{m-1}^{*}(x) d x \\
=\frac{\mathrm{Ch}_{n+1}^{*}(1) \mathrm{Ch}_{m}^{*}(1)-\mathrm{Ch}_{n+1}^{*} \mathrm{Ch}_{m}^{*}}{n+1}-\frac{m}{n+1} \frac{\mathrm{Ch}_{n+2}^{*}(1) \mathrm{Ch}_{m-1}^{*}(1)-\mathrm{Ch}_{n+2}^{*} \mathrm{Ch}_{m-1}^{*}}{n+2} \\
\quad+(-1)^{2} \frac{m}{n+1} \frac{m-1}{n+2} \int_{0}^{1} \mathrm{Ch}_{n+2}^{*}(x) \mathrm{Ch}_{m-2}^{*}(x) d x
\end{aligned}
$$


and

$$
\begin{aligned}
& \int_{0}^{1} \mathrm{Ch}_{n+m-1}^{*}(x) \mathrm{Ch}_{1}^{*}(x) d x \\
& \quad=\frac{\mathrm{Ch}_{n+m}^{*}(1) \mathrm{Ch}_{1}^{*}(1)-\mathrm{Ch}_{n+m}^{*} \mathrm{Ch}_{1}^{*}}{n+m}-\frac{1}{n+m} \int_{0}^{1} \mathrm{Ch}_{n+m}^{*}(x) \mathrm{Ch}_{0}^{*}(x) d x \\
& \quad=\frac{\mathrm{Ch}_{n+m}^{*}(1) \mathrm{Ch}_{1}^{*}(1)-\mathrm{Ch}_{n+m}^{*} \mathrm{Ch}_{1}^{*}}{n+m}-\frac{1}{n+m} \frac{\mathrm{Ch}_{n+m+1}^{*}(1)-\mathrm{Ch}_{n+m+1}^{*}}{n+m+1}
\end{aligned}
$$

By (30) with $x=0$ we get

$$
\begin{aligned}
& \int_{0}^{1} \mathrm{Ch}_{n}^{*}(x) \mathrm{Ch}_{m}^{*}(x) d x \\
& \quad=\sum_{j=0}^{m}\left(\begin{array}{c}
m \\
j
\end{array}\right) \mathrm{Ch}_{j}^{*} \int_{0}^{1} x^{m-j} \mathrm{Ch}_{m}^{*}(x) d x \\
& \quad=\sum_{j=0}^{m}\left(\begin{array}{c}
m \\
j
\end{array}\right) \mathrm{Ch}_{j}^{*} \sum_{l=0}^{m-j}(-1)^{m-j-l} \frac{(m-j)\left(\begin{array}{c}
m-j \\
l
\end{array}\right)}{(2(m-j)-l+1)\left(\begin{array}{c}
2(m-j)-l \\
m-j
\end{array}\right)} \mathrm{Ch}_{l}^{*}(1) \\
& \quad=\sum_{j=0}^{m} \sum_{l=0}^{m-j}\left(\begin{array}{c}
m \\
j
\end{array}\right)(-1)^{m-j-l} \frac{(m-j)\left(\begin{array}{c}
m-j \\
l
\end{array}\right)}{(2(m-j)-l+1)\left(\begin{array}{c}
2(m-j)-l \\
m-j
\end{array}\right)} \mathrm{Ch}_{j}^{*} \mathrm{Ch}_{l}^{*}(1) .
\end{aligned}
$$

By (37), continuing the process in (35), we obtain the following theorem.

Theorem 8 For $n \in \mathbb{N}$, we have

$$
\begin{aligned}
\sum_{j=0}^{m} \sum_{l=0}^{m-j}\left(\begin{array}{c}
m \\
j
\end{array}\right)(-1)^{m-j-l} \frac{(m-j)\left(\begin{array}{c}
m-j \\
l
\end{array}\right)}{(2(m-j)-l+1)\left(\begin{array}{c}
2(m-j)-l \\
m-j
\end{array}\right)} \mathrm{Ch}_{j}^{*} \mathrm{Ch}_{l}^{*}(1) \\
=\frac{\mathrm{Ch}_{n+1}^{*}(1) \mathrm{Ch}_{m}^{*}(1)-\mathrm{Ch}_{n+1}^{*} \mathrm{Ch}_{m}^{*}}{n+1} \\
\quad+\sum_{k=1}^{m-1}(-1)^{k} \frac{m(m-1) \cdots(m-k+1)}{(n+1)(n+2) \cdots(n+k+1)} \\
\quad \times\left(\mathrm{Ch}_{n+k+1}^{*}(1) \mathrm{Ch}_{m-k}^{*}(1)-\mathrm{Ch}_{n+k+1}^{*} \mathrm{Ch}_{m-k}^{*}\right) \\
\quad+(-1)^{m} \frac{m !}{(n+m+1)_{m+1}}\left(\mathrm{Ch}_{n+m+1}^{*}(1)-\mathrm{Ch}_{n+m+1}^{*}\right) .
\end{aligned}
$$

\section{Remarks}

In this section, by using the fermionic $p$-adic integral on $\mathbb{Z}_{p}$, we derive some identities for Changhee polynomials, Stirling numbers of the first kind, and Euler numbers. By (2) we note that

$$
\begin{aligned}
\frac{2}{2+t} e^{x t} & =\int_{\mathbb{Z}_{p}}(1+t)^{y} e^{x t} d \mu_{-1}(y) \\
& =\int_{\mathbb{Z}_{p}} e^{y \log (1+t)+x t} d \mu_{-1}(y)
\end{aligned}
$$


and

$$
\begin{aligned}
e^{x t} e^{y \log (1+t)} & =\left(\sum_{m=0}^{\infty} x^{m} \frac{t^{m}}{m !}\right)\left(\sum_{l=0}^{\infty} \frac{y^{l}(\log (1+t))^{l}}{l !}\right) \\
& =\left(\sum_{m=0}^{\infty} x^{m} \frac{t^{m}}{m !}\right)\left(\sum_{l=0}^{\infty} y^{l} \sum_{k=l}^{\infty} S_{1}(k, l) \frac{t^{k}}{k !}\right) \\
& =\left(\sum_{m=0}^{\infty} x^{m} \frac{t^{m}}{m !}\right)\left(\sum_{k=0}^{\infty} \sum_{l=0}^{k} y^{l} S_{1}(k, l) \frac{t^{k}}{k !}\right) \\
& =\sum_{n=0}^{\infty}\left(\sum_{k=0}^{n} \sum_{l=0}^{k}\left(\begin{array}{l}
n \\
k
\end{array}\right) x^{n-k} y^{l} S_{1}(k, l)\right) \frac{t^{n}}{n !} .
\end{aligned}
$$

Thus, by (39) and (40) we have

$$
\begin{aligned}
\sum_{n=0}^{\infty} \operatorname{Ch}_{n}^{*}(x) \frac{t^{n}}{n !} & =\int_{\mathbb{Z}_{p}} e^{y \log (1+t)} e^{x t} d \mu_{-1}(y) \\
& =\sum_{n=0}^{\infty}\left(\sum_{k=0}^{n} \sum_{l=0}^{k}\left(\begin{array}{l}
n \\
k
\end{array}\right) x^{n-k} \int_{\mathbb{Z}_{p}} y^{l} d \mu_{-1}(y) S_{1}(k, l)\right) \frac{t^{n}}{n !} \\
& =\sum_{n=0}^{\infty}\left(\sum_{k=0}^{n} \sum_{l=0}^{k}\left(\begin{array}{l}
n \\
k
\end{array}\right) x^{n-k} E_{l} S_{1}(k, l)\right) \frac{t^{n}}{n !} .
\end{aligned}
$$

From (41) we have the following theorem.

Theorem 9 For $n \in \mathbb{N}$, we have

$$
\mathrm{Ch}_{n}^{*}(x)=\sum_{k=0}^{n} \sum_{l=0}^{k}\left(\begin{array}{l}
n \\
k
\end{array}\right) x^{n-k} E_{l} S_{1}(k, l) .
$$

\section{Competing interests}

The authors declare that they have no competing interests.

\section{Authors' contributions}

All authors contributed equally to this work. All authors read and approved the final manuscript.

\section{Author details}

' Division of Mathematics and Informational Statistics, Nanoscale Science and Technology Institute, Wonkwang University, Iksan, 570-749, Republic of Korea. ${ }^{2}$ Graduate School of Education, Konkuk University, Seoul, 143-701, Republic of Korea. ${ }^{3}$ Department of Applied Mathematics, Pukyung National University, Busan, 608-737, Republic of Korea. ${ }^{4}$ Department of Mathematics Education, Konkuk University, Seoul, 143-701, Republic of Korea. ${ }^{5}$ Department of Mathematics, Kwangwoon University, Seoul, 139-701, Republic of Korea.

\section{Acknowledgements}

This paper was supported by Wonkwang University in 2015.

Received: 10 February 2016 Accepted: 17 May 2016 Published online: 21 June 2016

\section{References}

1. Bayad, A, Kim, T: Identities for the Bernoulli, the Euler and the Genocchi numbers and polynomials. Adv. Stud. Contemp. Math. (Kyungshang) 20(2), 247-253 (2010)

2. Bayad, A, Kim, T: Identities involving values of Bernstein, $q$-Bernoulli, and $q$-Euler polynomials. Russ. J. Math. Phys. 18(2), 133-143 (2011)

3. Carlitz, L: Degenerate Stirling, Bernoulli and Eulerian numbers. Util. Math. 15, 51-88 (1979) 
4. Kim, BM, Jang, LC: A note on the von Staudt-Clausen's theorem for the weighted $q$-Genocchi numbers. Adv. Differ. Equ. 2015, 4 (2015)

5. Kim, DS, Kim, T: Some identities of degenerate Euler polynomials arising from $p$-adic fermionic integrals on $\mathbb{Z}_{p}$. Integral Transforms Spec. Funct. 26(4), 295-302 (2015)

6. Kim, DS, Kim, T: Some identities of degenerate special polynomials. Open Math. 13, 380-389 (2015)

7. Kim, DS, Kim, T, Dolgy, DV: Degenerate q-Euler polynomials. Adv. Differ. Equ. 2015, 246 (2015)

8. Kim, DS, Kim, T: Some identities of Bell polynomials. Sci. China Math. 58(10), 1-10 (2015)

9. Kim, T: Note on the Euler numbers and polynomials. Adv. Stud. Contemp. Math. (Kyungshang) 17(2), 131-136 (2008)

10. Kim, T: Some properties on the integral of the product of several Euler polynomials. Quaest. Math. 38(4), 553-562 (2015)

11. Kim, T: Degenerate Euler zeta function. Russ. J. Math. Phys. 22(4), 469-472 (2015)

12. Kim, T: On the multiple $q$-Genocchi and Euler numbers. Russ. J. Math. Phys. 15(4), 481-486 (2008)

13. Kim, T, Mansour, T: Umbral calculus associated with Frobenius-type Eulerian polynomials. Russ. J. Math. Phys. 21(4), 484-493 (2008)

14. Kim, T: New approach to $q$-Euler, Genocchi numbers and their interpolation functions. Adv. Stud. Contemp. Math. (Kyungshang) 18(2), 105-112 (2009)

15. Kim, T: On Euler-Barnes multiple zeta functions. Russ. J. Math. Phys. 10(3), 261-267 (2003)

16. Kim, T: A study on the $q$-Euler numbers and the fermionic $q$-integral of the product of several type $q$-Bernstein polynomials on $\mathbb{Z}_{p}$. Adv. Stud. Contemp. Math. (Kyungshang) 23(1), 5-11 (2013)

17. Kim, T, Kim, DS: A note on nonlinear Changhee differential equations. Russ. J. Math. Phys. 23(1), 1-5 (2016)

18. Wang, NL, Li, H: Some identities on the higher-order Daehee and Changhee numbers. Pure Appl. Math. J. 5, 33-37 (2015)

19. Yilmaz Yasar, B, Özarslan, MA: Frobenius-Euler and Frobenius-Genocchi polynomials and their differential equations. New Trends Math. Sci. 3(2), 172-180 (2015)

20. Lim, D, Qi, F: On the Appell type $\lambda$-Changhee polynomials. J. Nonlinear Sci. Appl. 9, 1872-1876 (2016)

21. Kim, T, Park, JW, Seo, JJ: A note on $\lambda$-zeta function. Glob. J. Pure Appl. Math. 11(5), 3501-3506 (2015)

22. Adelberg, A: A finite difference approach to degenerate Bernoulli and Stirling polynomials. Discrete Math. 140(1-3), 1-21 (1995)

23. Carlitz, L: A degenerate Staudt-Clausen theorem. Arch. Math. (Basel) 7, 28-33 (1956)

24. Gaboury, S, Tremblay, R, Fugère, B-J: Some explicit formulas for certain new classes of Bernoulli, Euler and Genocchi polynomials. Proc. Jangjeon Math. Soc. 17(1), 115-123 (2014)

25. Howard, FT: Explicit formulas for degenerate Bernoulli numbers. Discrete Math. 162(1-3), 175-185 (1996)

26. Liu, GD: Degenerate Bernoulli numbers and polynomials of higher order. J. Math. (Wuhan) 25(3), 283-288 (2005) (in Chinese)

27. Mahmudov, NI, Akkeles, A, Öneren, A: On two dimensional q-Bernoulli and q-Genocchi polynomials: properties and location of zeros. J. Comput. Anal. Appl. 18(5), 834-843 (2015)

28. Kwon, JK: A note on weighted Boole polynomials. Glob. J. Pure Appl. Math. 11(4), 2055-2063 (2015)

\section{Submit your manuscript to a SpringerOpen ${ }^{\circ}$ journal and benefit from:}

- Convenient online submission

Rigorous peer review

- Immediate publication on acceptance

- Open access: articles freely available online

- High visibility within the field

- Retaining the copyright to your article 